\title{
Relativistic Treatment of Spinless Particles Subject to Modified Scarf II Potential
}

\author{
Akaninyene Daniel Antia ${ }^{1, ~ *, ~ I t a ~ O k o n ~ A k p a n ², ~ A k a n i n y e n e ~ O k o n ~ A k a n k p o ' ~}$ \\ ${ }^{1}$ Department of Physics, Faculty of Science, University of Uyo, Uyo, Akwa Ibom State, Nigeria \\ ${ }^{2}$ Department of Physics, Faculty of Science, University of Calabar, Calabar, Cross Rivers State, Nigeria
}

Email address:

antiacauchy@yahoo.com (A. D. Antia), akaninyeneantia@uniuyo.edu.ng (A. D. Antia)

\section{To cite this article:}

Akaninyene Daniel Antia, Ita Okon Akpan, Akaninyene Okon Akankpo. Relativistic Treatment of Spinless Particles Subject to Modified Scarf II Potential. International Journal of High Energy Physics. Vol. 2, No. 4, 2015, pp. 50-55. doi: 10.11648/j.ijhep.20150204.12

\begin{abstract}
We employ the parametric generalization of Nikiforov-Uvarov method to obtain the bound state solutions the relativistic Klein-Gordon equation under equal scalar and vector modified Scarf II potential. The energy eigenvalues and the corresponding wave functions expressed in term of Jacobi polynomial are equally obtained. Our results will have many applications in many branches of physics especially nuclear physics where it could be used in describing nuclei interactions .For further guide to interested readers, we have also provided numerical data which discuses the energy spectra.
\end{abstract}

Keywords: Klein-Gordon Equation, Modified Scarf II Potential, Bound State Solution, Nikiforov-Uvarov Method

\section{Introduction}

It is a well known fact that when a particle is in a strong potential field, the relativistic effect must be considered, leading to the relativistic quantum mechanical description of such particle [1-5]. In the relativistic limit, the particle motions are commonly described using either the KleinGordon equation or the Dirac equation [1,3] depending on the spin character of the particle. The spin-zero particles for example, the mesons are described by the Klein-Gordon equation. On the other hand, the half integral spin particles such as electron are described satisfactorily by the Dirac equation. One of the interesting problems in nuclear and high energy physics is to obtain exact or approximate solutions of the Klein-Gordon and Dirac equations. In recent years, many studies have been carried out to explore the relativistic energy eigenvalues and the corresponding wave functions of the Klein-Gordon and Dirac equations [6-9]. These relativistic equations contain two objects: the vector potential $V(r)$ and the scalar potential $S(r)$.

The Klein-Gordon equation with the vector and scalar potentials can be written as follows

$$
\left[-\left(i \frac{\partial}{\partial t}-V(r)\right)^{2}-\nabla^{2}+(S(r)+M)^{2}\right] \psi(r, \theta, \varphi)=0
$$

where $M$ is the rest mass, $i \frac{\partial}{\partial t}=E$ is the energy eigenvalues, $V(r)$ and $S(r)$ are the vector and scalar potentials respectively. Many researchers have studied the bound states of the Klein-Gordon and the Dirac equation with mixed potentials by assuming equal scalar and vector potentials. For example, these investigations have employed WoodsSaxon potential [10], Hartmann potential [11], anharmonic oscillator potential [2], ring shape pseudoharmonic potential [12], Kratzer potential [13, 14] and Poschl-Teller and Rosen Morse potential [15]. Different methods such as the asymptotic iteration method (AIM) [16], supermmetry quantum mechanics [17], Nikiforov-Uvarov (NU) method $[10,18]$ and others have been used to solve the differential equations arising from these considerations.

However, exact analytical solutions of the Klein-Gordon equations are possible only in the s-wave case with the angular momentum quantum number $l=0$ for some typical exponential type potential models [19]. Conversely, when $l \neq 0$, one can only solve approximately the KleinGordon equation and the Dirac equation for some potentials using a suitable approximation scheme [20].

The bound state energy spectra and the corresponding wave functions for the scarf - type potential have been investigated by a variety of method. The main aim of this paper is to obtain the energy eigenvalues and corresponding wave functions of the Klein-Gordon equation with modified Scarf II potential using a new improved approximation scheme [21] to deal with the centrifugal term under the frame work of Nikiforov-Uvarov method. This potential has many applications in nuclear physics and in describing nuclei interactions. 


\section{Nikiforov-Uvarov Method and its Parametric Form}

The Nikiforov-Uvarov method (NU) [22] was proposed to solve the second order linear differential equation by reducing it to a generalized hypergeometric-type of the form

$$
\psi^{\prime \prime}(s)+\frac{\bar{\tau}(s)}{\sigma(s)} \psi^{\prime}(x)+\frac{\bar{\sigma}(s)}{\sigma^{2}(s)} \psi(s)=0,
$$

where the prime denote the differentiation with respect to $s, \sigma(s)$ and $\bar{\sigma}(s)$ are polynomials at most second degree, $\bar{\tau}(s)$ is a first degree polynomial. In order to find the exact solution to equation (2), we set the wave function as

$$
\psi(x)=\phi(s) \chi(s)
$$

Substituting Eq. (3) into Eq. (2) reduces Eq. (2) into a hypergeometric type equation

$$
\sigma(s) \chi_{n}^{\prime \prime}(s)+\tau(s) \chi_{n}^{\prime}(s)+\lambda \chi_{n}(s)=0
$$

where the wave function $\phi(s)$ is defined as a logarithmic derivative in the following forms and its solutions can be obtained from

$$
\frac{\phi^{\prime}(s)}{\phi(s)}=\frac{\pi(s)}{\sigma(s)}
$$

where $\pi(s)$ is at most first-degree polynomials.

The other part $\chi(s)$ is the hypegeometric type function whose polynomial solutions are given by the Rodriques relation

$$
\chi_{n}(s)=\frac{B_{n}}{\rho(s)} \frac{d^{n}}{d s^{n}}\left[\sigma^{n}(s) \rho(s)\right]
$$

where $B_{n}$ is the normalization constant and the weight function $\rho(s)$ satisfy the condition

$$
\frac{d}{d s}(\sigma(s) \rho(s))=\tau(s) \rho(s)
$$

The required $\pi(s)$ and $\lambda$ for the NU method are defined as

$$
\pi(s)=\frac{\sigma^{\prime}(s)-\bar{\tau}}{2} \pm \sqrt{\left(\frac{\sigma^{\prime}(s)^{\prime}-\bar{\tau}}{2}\right)^{2}-\bar{\sigma}(s)+k \sigma(s)}
$$

and

$$
\lambda=k+\pi^{\prime}(s)
$$

respectively. Therefore, the determination of $k$ in Eq. (8) is the necessary condition in the calculation of $\pi(s)$ for which the determinant of the square root in Eq. (8) is set to zero. The eigenvalues equation defined in Eq. (9) takes the form

$\lambda=\lambda_{n}=-n \frac{d \tau}{d s}-\frac{1}{2} n(n-1) \frac{d^{2} \sigma}{d s^{2}}, n=0,1,2 \ldots$

where

$$
\tau(s)=\bar{\tau}(s)+2 \pi(s)
$$

and its derivative is negative which in the necessary condition for bound state solutions. The energy eigenvalues equation is obtained by comparing Eqs. (9) and (10).

Now, in order to clarify the parametric generalization of the NU method [23], let us take the following Schrödinger like equation written for any potential:

$$
\psi^{\prime \prime}(s)+\frac{c_{1}-c_{2} s}{s\left(1-c_{3} s\right)} \psi^{\prime}(s)+\frac{1}{s^{2}\left(1-c_{3} s\right)^{2}}\left[-\xi_{1} s^{2}+\xi_{2} s-\xi_{3}\right] \psi(s)=0 .
$$

Comparing Eq. (12) with Eq. (2), we obtain the following parametric polynomials.

$\bar{\tau}(s)=c_{1}-c_{2} s, \sigma(s)=s\left(1-c_{3} s\right), \bar{\sigma}(s)=-\xi_{1} s^{2}+\xi_{2} s-\xi_{3}$

Substituting Eqs. (13) into Eq. (8), we obtain

$$
\pi(s)=c_{4}-c_{5} s \pm\left[\left(c_{6}-c_{3} k_{ \pm}\right) s^{2}\left(c_{7}+k_{ \pm}\right) s+c_{8}\right]^{\frac{1}{2}}
$$

where

$$
\begin{gathered}
c_{4}=\frac{1}{2}\left(1-c_{1}\right), c_{5}=\frac{1}{2}\left(c_{2}-2 c_{3}\right), c_{6}=c_{5}^{2}+\xi_{1} \\
c_{7}=2 c_{4} c_{5}-\xi_{2}, c_{8}=c_{4}^{2}+\xi_{3}
\end{gathered}
$$

We obtain the parametric $k_{ \pm}$from the condition that the function under the square root should be the square of a polynomial,

$$
k_{ \pm}=-\left(c_{7}+2 c_{3} c_{8}\right) \pm 2 \sqrt{c_{8} c_{9}}
$$

where $c_{9}=c_{3} c_{7}+c_{3}^{2} c_{8}+c_{6}$. The function $\pi(s)$ in Eq. (4) becomes

$$
\pi(s)=c_{4}+c_{5} s-\left[\left(\sqrt{c_{9}}+c_{3} \sqrt{c_{8}}\right) s-\sqrt{c_{8}}\right]
$$

for the negative $k_{-}$value, we have

$$
k_{-}=-\left(c_{7}+2 c_{3} c_{8}\right)-2 \sqrt{c_{8} c_{9}}
$$

thus, from the relation $\tau(s)=\bar{\tau}(s)+2 \pi(s)$, we have

$$
\tau(s)=c_{1}+2 c_{4}-\left(c_{2}-2 c_{5}\right) s-2\left[\left(\sqrt{c_{9}}+c_{3} \sqrt{c_{8}}\right) s-\sqrt{c_{8}}\right]
$$

Thus the necessary condition for the bound state solution is imposed by the relation,

$$
\tau^{\prime}(s)=-2 c_{3}-2\left[\left(\sqrt{c_{9}}+c_{3} \sqrt{c_{8}}\right)\right]<0 .
$$

Equating Eq. (9) with Eq. (10) and making use of Eqs. (13), (17), (18) and (20) we obtain the parametric energy eigenvalue equation for potential under consideration as

$$
\begin{gathered}
\left(c_{2}-c_{3}\right) n+c_{3} n^{2}-(2 n+1) c_{5}+(2 n+1)\left[\sqrt{c_{9}}+\right. \\
c 3 c 8+c 7+2 c 3 c 8+2 c 8 c 9=0
\end{gathered}
$$

the weight function $\rho(s)$ is obtain as

$$
\rho(s)=s^{c_{10}}\left(1-c_{3} s\right)^{c_{11}}
$$

and together with Eq. (6), we obtain

$$
\chi_{n}(s)=\rho_{n}^{\left(c_{10}, c_{11}\right)}\left(1-2 c_{3} s\right), c_{10}>1, c_{11}>-1,
$$

where 


$$
\begin{aligned}
& c_{10}=c_{1}+2 c_{4}+2 \sqrt{c_{8}} \\
& c_{11}=1-c_{1}-2 c_{4}+\frac{2}{c_{3}} \sqrt{c_{9}}
\end{aligned}
$$

and $P_{n}^{(a, b)}\left(1-2 c_{3} s\right)$ are the Jacobi polynomials. Also, the second part of the wave functions can be found from Eq. (5) as

$$
\phi(s)=s^{c_{12}}\left(1-c_{3} s\right)^{c_{13}}, c_{12}>0, c_{13}>0,
$$

where

$$
\begin{gathered}
c_{12}=c_{4}+\sqrt{c_{8}} \\
c_{13}=--c_{4}+\frac{1}{c_{3}}\left(\sqrt{c_{9}}-c_{5}\right)
\end{gathered}
$$

Hence, the wave function has the form

$$
\psi_{n}(s)=N_{n} s^{c_{12}}\left(1-c_{3} s\right)^{c_{13}} P_{n}^{\left(c_{10}, c_{11}\right)}\left(1-2 c_{3} s\right)
$$

where $N_{n}$ is the normalization constant.

\section{Factorization Method for Klein- Gordon Equation}

The three dimensional Klein-Gordon equation with the mixed vector and scalar potentials can be written as [24]

$$
\left[\nabla^{2}+(V(r)-E)^{2}-(S(r)+M)^{2}\right] \psi(r, \theta, \varphi)=0,
$$

where $M$ is the rest mass, $E$ is the relativistic energy, $S(r)$ and $V(r)$ are the scalar and vector potentials respectively. $\nabla^{2}$ is the Laplace operator, $c$ is the speed of light and $\hbar$ is the reduced planck's constant which have been set to unity. In spherical coordinates, the Klein-Gordon equation for a particle in the present of a modified Scarf II potential $V(r)$ becomes

$$
\begin{array}{r}
{\left[\frac{1}{r^{2}} \frac{\partial}{\partial r}+\left(r^{2} \frac{\partial}{\partial r}\right)+\frac{1}{r^{2} \sin \theta} \frac{\partial}{\partial \theta}\left(\sin \theta \frac{\partial}{\partial r}\right)+\frac{1}{r^{2} \sin ^{2} \theta} \frac{\partial^{2}}{\partial \varphi^{2}}-\right.} \\
2 E V r+M S r+\quad V 2 r+S 2 r+E 2-M 2 \psi r, \theta, \varphi=0
\end{array}
$$

If one assigns the corresponding spherical total wave function as

$$
\psi(r, \theta, \varphi)=\frac{R(r)}{r} Y(\theta, \varphi)
$$

Where

$$
Y(\theta, \varphi)=\Theta(\theta) \Phi(\varphi)
$$

Thus, the wave function in Eq. (31) is separated into variables and the following set of equations are obtained

$$
\begin{gathered}
\frac{d^{2} R(r)}{d r^{2}}+\left[E^{2}-M^{2}-2(E V(r)+M S(r))+V^{2}(r)+\right. \\
\left.S^{2}(r)-\frac{\lambda}{r^{2}}\right] R(r)=0 \\
\frac{d^{2} \Theta(\theta)}{d \theta^{2}}+\cot \theta \frac{d \Theta(\theta)}{d \theta}+\left[\lambda-\frac{m^{2}}{\sin ^{2} \theta}\right] \Theta(\theta)=0 \\
\frac{d^{2} \Phi(\varphi)}{d \varphi^{2}}+m^{2} \Phi(\varphi)=0
\end{gathered}
$$

where $m^{2}$ and $\lambda=l(l+1)$ are the separation constants.

The solutions of equation (35) and (36) are well known [25].

\section{Solutions of the Radial Klein-Gordon Equation}

The modified scarf II potential is defined in the form [26]

$$
V(r)=-\frac{1}{2}\left(\gamma^{2}+\beta^{2}-\frac{1}{2}\right) \operatorname{sech}^{2} \alpha r+\frac{1}{2}\left(\gamma^{2}-\beta^{2}\right) \tanh ^{2} \alpha r
$$

where $\gamma, \beta$ are potential parameters and $\alpha$ is range of the potential. We have shown in Fig.1 and Fig. 2, the plot of the Scarf II potential as a function of $\mathrm{r}$ for $\alpha=1,2,3,4, \beta=$ $0.25, \gamma=0.1$ and $\alpha=1,2,3,4, \beta=0.9, \gamma=0.1$ respectively. The radial equation of the Klein-Gordon equation for the special case $V(r)=S(r)$ is obtained as

$$
\begin{aligned}
& \frac{d^{2} R(r)}{d r^{2}}+\left[E^{2}-M^{2}+\frac{1}{2}\left(\gamma^{2}+\beta^{2}-\frac{1}{2}\right)(E+M) \operatorname{sech}^{2} \alpha r-\right. \\
& \left.(E+M)\left(\gamma^{2}-\beta^{2}\right) \tanh ^{2} \alpha r-\frac{\lambda}{r^{2}}\right] R(r)=0 .
\end{aligned}
$$

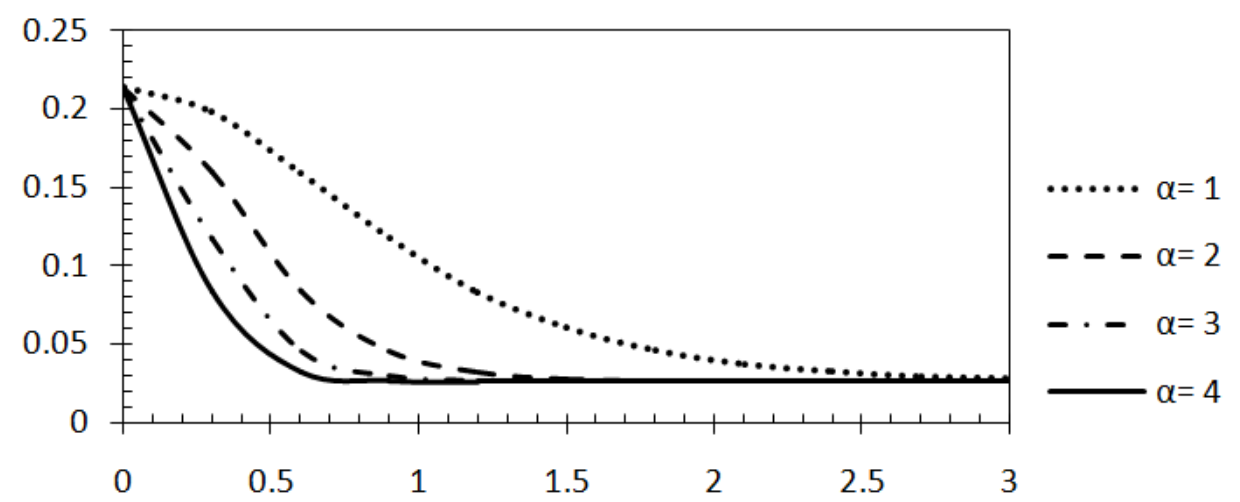

Fig. 1. Graph of modified Scarf II potential as a function of $r$ for various values of $\alpha=1,2,3$ and 4 with $\beta=0.25$ and $Y=0.1$.

Equation (38) cannot be solved analytically because of the centrifugal term. We now use the new improved approximation scheme [21] for the centrifugal term as 


$$
\frac{1}{r^{2}} \approx 4 \alpha^{2}\left[c_{o}+\frac{e^{-2 \alpha r}}{\left(1-e^{-2 \alpha r}\right)^{2}}\right]=4 \alpha^{2}\left[c_{o}+\frac{1}{4 \sinh ^{2} \alpha r}\right]
$$

where $c_{o}=\frac{1}{12}$ is an arbitrary dimensionless constant. In this study we set $c_{o}=0$, which reduces Eq. (39) to conventional approximation scheme [27].

Substituting Eq. (39) into Eq. (38), we obtain $\frac{d^{2} R(r)}{d r^{2}}+\left[\widetilde{E^{2}}+\widetilde{V_{1}} \operatorname{sech}^{2} \alpha r-\widetilde{V_{2}} \tanh ^{2} \alpha r-\frac{\alpha^{2} l(l+1)}{\sinh ^{2} \alpha r}\right] R(r)=0$,

where

$$
\left.\begin{array}{c}
\widetilde{E^{2}}=E^{2}-M^{2}, \widetilde{V_{1}}=(E+M)\left(\gamma^{2}+\beta^{2}-\frac{1}{2}\right) \\
\widetilde{V_{2}}=(E+M)\left(\gamma^{2}-\beta^{2}\right)
\end{array}\right\}
$$

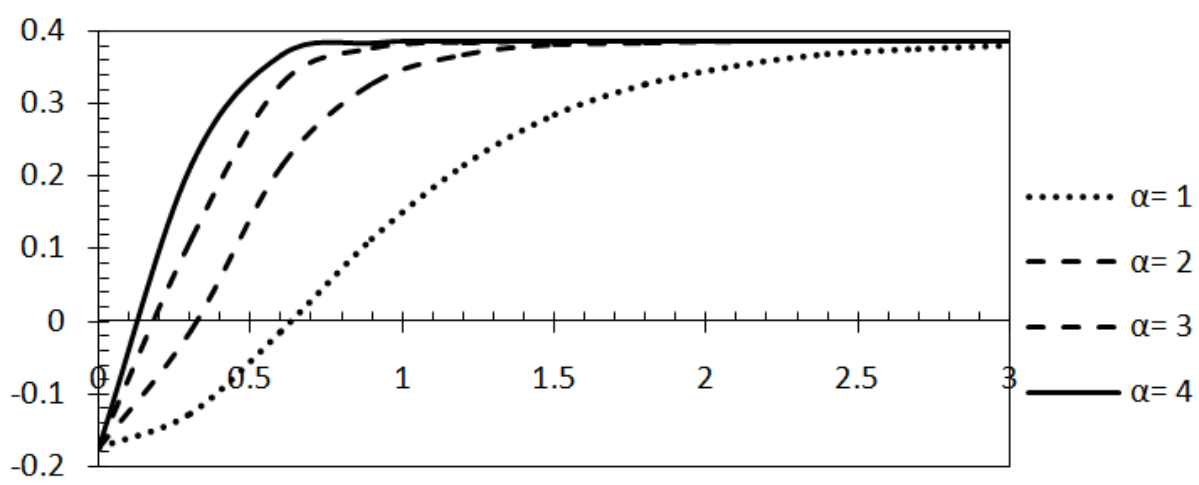

Fig. 2. Graph of modified Scarf II potential as function of $r$ for various values of $\alpha=1,2,3$ and 4 with $\beta=0.9$ and $Y=0.2$.

Introducing a new variable $\mathrm{s}=\sinh ^{2} \alpha r$ and substituting into Eq. (40), we have the following hypergeometric equation,

$$
\begin{gathered}
\frac{d^{2}}{d s^{2}}+\frac{\left(\frac{1}{2}+s\right)}{s(1+s)} \frac{d R(s)}{d s}+\frac{1}{s^{2}(1+s)^{2}}\left[-\left(\varepsilon^{2}+\Lambda^{2}\right) s^{2}\left(\delta^{2}-\varepsilon^{2}+\right.\right. \\
g 2 s-g 2 R s=0,
\end{gathered}
$$

where the following dimensionless quantities have been used in obtaining Eq. (42)

$$
-\varepsilon^{2}=\frac{\widetilde{E^{2}}}{4 \alpha^{2}}, \delta^{2}=\frac{\widetilde{V_{1}}}{4 \alpha^{2}}, \Lambda^{2}=\frac{\widetilde{V_{2}}}{4 \alpha^{2}}, g^{2}=\frac{l(l+1)}{4}
$$

By comparing Eq. (42) with Eq. (12), we obtain the parameter set

$$
\begin{gathered}
c_{1}=\frac{1}{2}, c_{2}=-1, c_{3}=-1 \\
\xi_{1}=\varepsilon^{2}+\wedge^{2}, \xi_{2}=\delta^{2}-\varepsilon^{2}+g^{2}, \xi_{3}=g^{2}
\end{gathered}
$$

$$
\pi(s)=\frac{1+2 s}{4} \pm\left\{\begin{aligned}
& \left(\sqrt{\frac{1}{16}+\delta^{2}+\Lambda^{2}}+\sqrt{\left.\frac{1}{16}+g^{2}\right) s+\sqrt{\frac{1}{16}+g^{2}}}\right. \\
\text { for } k_{+}= & -\left(\frac{1}{8}-\delta^{2}+\varepsilon^{2}-g^{2}\right)+2 \sqrt{\left(\frac{1}{16}+g^{2}\right)\left(\frac{1}{16}+\delta^{2}+\Lambda^{2}\right)} \\
& \left(\sqrt{\frac{1}{16}+\delta^{2}+\Lambda^{2}}-\sqrt{\left.\frac{1}{16}+g^{2}\right) s-\sqrt{\frac{1}{16}+g^{2}}}\right. \\
\text { for } k_{-}= & -\left(\frac{1}{8}-\delta^{2}+\varepsilon^{2}-g^{2}\right)-2 \sqrt{\left(\frac{1}{16}+g^{2}\right)\left(\frac{1}{16}+\delta^{2}+\Lambda^{2}\right)}
\end{aligned}\right.
$$

Also using Eq. (17), we find the following physical values

$$
\pi(s)=\frac{1+2 s}{4}-\left[\left(\sqrt{\frac{1}{16}+\delta^{2}+\Lambda^{2}}-\sqrt{\frac{1}{16}+g^{2}}\right) s-\sqrt{\frac{1}{16}+g^{2}}\right]
$$

Using Eqs. (18) and (44), we calculate the negative $k_{-}$required for the NU method as
Using Eqs.(14),(16) and (44),we obtain the $\pi(s)$ as 


$$
k_{-}=-\left(\frac{1}{8}-\delta^{2}+\varepsilon^{2}-g^{2}\right)-2 \sqrt{\left(\frac{1}{16}+g^{2}\right)\left(\frac{1}{16}+\delta^{2}+\Lambda^{2}\right)},
$$

also using Eq. (20), we obtain $\tau^{\prime}(s)$ as

$$
\tau^{\prime}(s)=-2\left[\sqrt{\frac{1}{16}+\delta^{2}+\Lambda^{2}}-\sqrt{\frac{1}{16}+g^{2}}-2\right]<0
$$

which is the essential condition for bound state solution.

Using Eq. (21), we obtain the energy equation

$$
\varepsilon^{2}=\delta^{2}+g^{2}+\frac{3}{8}+n(n+1)-(2 n+1)\left[\sqrt{\frac{1}{16}+\delta^{2}+\wedge^{2}}-\sqrt{\frac{1}{16}+g^{2}}\right]-2 \sqrt{\left(\frac{1}{16}+g^{2}\right)\left(\frac{1}{16}+\delta^{2}+\wedge^{2}\right)}
$$

Substituting Eq. (43) into (49), we obtain the energy eigenvalues of the system as

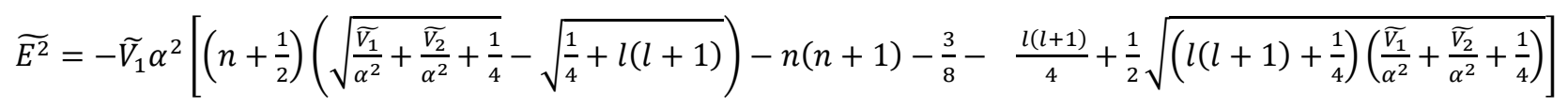

Table 1. Bound state energy of modified Scarf II potential for $m=1, \beta=0.1$ and $\gamma=0.5$.

\begin{tabular}{llll}
\hline $\mathbf{n}$ & $\boldsymbol{l}$ & Energy level for $\boldsymbol{\alpha}=\mathbf{0 . 0 5}$ & Energy level for $\boldsymbol{\alpha}=\mathbf{0 . 0 2}$ \\
\hline 0 & 0 & -0.9988833719 & -0.9998214143 \\
1 & 0 & -0.9808632976 & -0.9969601604 \\
2 & 0 & -0.9532660434 & -0.9926544837 \\
2 & 1 & -0.9403772913 & -0.9906754702 \\
3 & 0 & -0.9153260356 & -0.9868875282 \\
3 & 1 & -0.8970545708 & -0.9841738993 \\
3 & 2 & -0.8759417654 & -0.9810903687 \\
4 & 0 & -0.8658498872 & -0.9796363035 \\
4 & 1 & -0.8414572273 & -0.9761751685 \\
4 & 2 & -0.8137558567 & -0.9723369444 \\
4 & 3 & -0.7824411338 & -0.9681176407 \\
5 & 0 & -0.8029842902 & -0.9708712115 \\
5 & 1 & -0.7712911982 & -0.9666462166 \\
5 & 2 & -0.7355115429 & -0.9620351196 \\
5 & 3 & -0.6951073549 & -0.9570329614 \\
5 & 4 & -0.6493503753 & -0.9516342660 \\
\hline
\end{tabular}

The energy eigenvalues of this system is computed for $\mathrm{m}=1, \beta=0.1, \gamma=0.5$ for various values of potential range $\alpha=0.05$ and 0.02 as presented in table 1 . The weight function in Eq. (22) can be calculated as

$$
\rho(s)=s^{1+2 \sqrt{\frac{1}{16}+g^{2}}}(1+s)^{-2 \sqrt{\frac{1}{16}+\delta^{2}+\wedge^{2}}}
$$

which gives the first part of the wave function in Eq. (3) as

$$
\chi_{n}(s)=P_{n}^{(1+2 \mu, 2 v)}(1+2 s)
$$

where

$$
\mu=\sqrt{\frac{1}{16}+g^{2}} \text { and } v=-\sqrt{\frac{1}{16}+\delta^{2}+\wedge^{2}}
$$

Also, the second part of the wave function in Eq. (26) can be found as

$$
\phi(s)=s^{\frac{1}{4}+\mu}(1+s)^{\frac{1}{4}+v}
$$

Hence, the unnormalized wave function is obtained as $\psi_{n, l}(r)=N_{n}\left(\sinh ^{2} \alpha r\right)^{\left(\frac{1}{4}+\mu\right)}\left(\cosh ^{2} \alpha r\right)^{\frac{1}{4}+v} P_{n}^{(1+2 \mu, 2 v)}\left(1+2 \sinh ^{2} \alpha r\right),(54)$ where $N_{n}$ is the normalization constant.

\section{Conclusion}

We have obtained the energy spectra and the corresponding wave functions of the Klein-Gordon equation under equal scalar and vector modified Scarf II potential by using Nikiforov-Uvarov method and employing the improved approximation scheme to deal with the centrifugal term. We found a closed form of the energy eigenvalues for arbitrary $l$ state.The behaviours of the potential with the particle position, $r$ for various potential parameters are presented.The numerical data of our results are also presented as a guide to our readers. This system can be used to investigate the relativistic symmetries of the Dirac equation [28].

\section{References}

[1] L. Z. Yi, Y. F. Diao, J. Y. Liu, C. S. Jia (2004). Phys.Lett. A. $333,212$.

[2] G. F. Wei, C. Y. Long, Z. He, S. J. Qin and J.Zhao (2007). Phys. Scr. 76, 442.

[3] X. C.Zhang, Q. W. Lia, C. S. Jia, L. Z. Wang (2005). Phys. Lett. A. 340, 59 .

[4] W. A. Yahya, K. J. Oyewumi, C. O. Akoshile and T. T. Ibrahim (2010). J. Vect Relat. 5(3), 27.

[5] A. D.Antia, A. N.Ikot, E. E.Ituen and I.O.Akpan (2012). Sri Lankan Journal of Physics 13(1), 27-40.

[6] K. J. Oyewumi and C. O. Akoshile (2010). Eur. Phys. J. A 45, 311.

[7] A. N. Ikot, L. E. Akpabio and E. J. Uwah (2011) EJTP, 25(8), 225-232.

[8] A. N. Ikot, A. B. Udoimuk and L. E. Akpabio (2011). Am. J. Sci. Ind. Res. 2(2), 179-183. 
[9] H. Akcay, C. Tezcan (2009). Int. J. Mod. Phys C. 20(6), 931.

[10] C. Berkdemi, A. Berkdemir and R. Sever (2006). J. Phys. A : Math Gen. 399, 13455.

[11] A. desouza Dutra and M. Hott (2006). Phys Lett. A 356, 215.

[12] S. Ikhdair and R. Sever (2008). Int. J. Mod. Phys C 19, 1425.

[13] W.C. Qiang (2004). Chin. Phys. 13, 571.

[14] W. C. Qiang (2003). Chin. Phys. 12, 1054.

[15] A.D. Alhaidari (2001). J. Phys. A: Math. Gen. 34, 9827.

[16] H. Ciftci, R. L. Hall and N. Saad (2003). J. Phys. A. 36, 11807.

[17] C. S. Jia, P. Gao and X. L. Peng (2006). J. Phys. A. Math. Gen. $39,7737$.

[18] Y. F. Cheng and T. Q. Dai (2007). Chin. J. Phys. 45, 480.

[19] Y. F. Diao, L. Z. Yi and C. S. Jia (2004). Phys. Lett A 332, 157.
[20] Y. Xu, S. He and C. S. Jia (2010). Phys Scr. 81, 045001.

[21] C. S. Jia,T. Chen and L. G. Cui (2009). Phys Lett. A 373, 1621.

[22] A. F. Nikiforov and V. B. Uvarov (1988). Special Functions of Mathematical Physics. (Basel, Birkhauser).

[23] C. Tezcan and R. Sever (2008). Int. J. Theor. Phys 47, 1471.

[24] W. C. Qiang and S. H. Dong (2008). Phys. Lett. A 372, 4789.

[25] S. Flugge, Practical Quantum Mechanics (1974). (Springer, Berlin).

[26] G. Levai (2011). Int. J. Theor. Phys. 50, 997.

[27] R. I. Greene and C. Aldrich (1976). Phys. Rev. A 14, 2363.

[28] A. N. Ikot, E. Maghsoodi, A. D. Antia, H. Hassanabadi and S. Zarrinkamar (2015). Arab. J. Sci. Eng. DOI 10.1007/s13369015-1602-4. 